\title{
Epidemiología del cáncer en adolescentes
}

\author{
María Luisa C uevas-U rióstegui, MC,(1) Miguel A ngel Villasís-Keever, MC, M en C, , $^{(1)}$ \\ Arturo Fajardo-Gutiérrez, MC, M en C.(1)
}

\section{Cuevas-Urióstegui ML,Villasís-Keever MA, Fajardo-Gutiérrez A. Epidemiología del cáncer en adolescentes. Salud Publica Mex 2003;45 supl 1:S115-S123.}

\section{Resumen}

Objetivo. Revisar los artículos publicados sobre la epidemiología del cáncer en adolescentes en el ámbito mundial. Material y métodos Se revisó la literatura médica (Medline) nacional e internacional para un periodo de 15 años (1985-1999). Se calcularon frecuencias. Resultados. En general se conoce poco de la epidemiología del cáncer en adolescentes porque es difícil el registro de los casos que se presentan en el grupo de 12 a 18 años. Como adolescentes se ha tomado al grupo de 10 a 140 el de 15 a 19 años de edad, en donde este último incluye mejor los casos que se presentan en esta edad. La incidencia es mayor para el segundo grupo (117.3 y 202.2 [tasas $\times 10^{6}$ ] respectivamente). Las principales neoplasias para el grupo de 15 a 19 años de edad fueron los tumores del sistema nervioso central (TSN C), las leucemias, los linfomas, los tumores óseos, los de células germinales y carcinomas. La incidencia es mayor en el sexo masculino y la raza blanca. Existe una tendencia al incremento. La mortalidad en adolescentes ha disminuido (2.0 a 3.2\% por año en Estados U nidos deA mérica [EUA]). La so brevida se ha incrementado en todos los tipos de neoplasias. En México no se cuenta con datos de incidencia para el grupo de 15 a 19 años de edad; sólo se tiene para el de 10 a 14 años; las principales neoplasias son leucemias, linfomas, tumores óseos y los TSN C, con tasas por $10^{6}$ de $41.9,29.9,12.0$ y 10.0 respectivamente. La tasa de mortalidad por cáncer para el periodo de 1990 a 1994 fue de 64.1, y las principales causas de muerte fueron las leucemias, los TSN C y los linfo mas. Conclusiones Es recomendable, en

\author{
Cuevas-Urióstegui ML,Villasís-Keever MA, \\ Fajardo-Gutiérrez A. \\ The epidemiology of cancer in adolescents \\ Salud Publica Mex 2003;45 suppl 1:S115-S123.
}

\begin{abstract}
A bstract
Objective. To review the international literature on adolescent cancer epidemiology. Material and Methods.The Medline database was searched to obtain all papers on adolescent cancer epidemiology published during a period of 15 years (1985-1999).Time, place, and per son variables were analyzed, as well as their relationships. Results Few papers on adolescent cancer epidemiology were found, likely due to the difficulty in registering cancer cases in the 12 to 18 year-old group. Either the 10-14 or the 15-19 year-old group has been analyzed in different studies; but the latter is more representative of teenagers. A higher incidence rate was found in this group (117.3 and $202.2 \times 10^{6}$ respectively). The main malignancies in the $15-19$ year-old group were central nervous system tumors (CN ST), leukemias, lymphomas, bone tumors, germ-cell tumors, and carcinomas. Incidence rates were higher in males and whites; an increasing trend was observed. Cancer mortality in this group is decreasing in the United States $(2.0$ or $3.2 \%$ per year). Survival is increasing in all groups of neoplasms. $\mathrm{N} O$ data were available on cancer incidence in the 15-19 year-old group in Mexico; data were available only for the 10-14 year-old group.The main neoplasms are leukemias, lympho mas, bone tumors, and CN ST (rates were 41.9, 29.9, 12.0, and $10.0 \mathrm{x}$ $10^{6}$, respectively). The mortality rate for the 1990-1994 period was 64.1 and the main neoplasms were leukemias, CNST, and lymphomas. Conclusions More studies are needed to address the descriptive epidemiology of cancer in teenagers.
\end{abstract}

(1) Unidad de Investigación Médica en Epidemiología Clínica, Hospital de Pediatría, Centro Médico N acional Siglo XXI. Instituto Mexicano del Seguro Social, México, D.F., México.

Fecha de recibido: 18 de abril de 2001 - Fecha de aprobado: 21 de noviembre de 2001

Solicitud de sobretiros: Dr. Arturo Fajardo-Gutiérrez. Unidad de Investigación Médica en Epidemiología Clínica, Hospital de Pediatría, Centro Médico Nacional Siglo XXI, IMSS. Av. Cuauhtémoc 330, colonia D octores, 06720 México, D.F., México.

Correo electrónico: afajardo@ servidor.unam.mx 
general, y en particular, en nuestro país, el desarrollo de proyectos dirigidos a conocer y difundir la epidemiología descriptiva del cáncer en adolescentes.

Palabras clave: ado lescencia; neo plasmas; incidencia; mortalidad
Key words: adolescence; neoplasms; incidence; mortality
U no de los principales problemas para el estudio de la epidemiología del cáncer en adolescentes, es la dificultad del registro de los casos que se presentan en este grupo de edad. Y aunque la Organización Mundial de la Salud $(\mathrm{OMS})^{1}$ propone que la adolescencia se considere entre los 10 y 19 años, estableciendo que para fines estadísticos se evalúe al grupo de 10 a 14 años de edad como adolescencia temprana, y al de 15 a 19 años de edad como adolescencia tardía, es difícil obtener los casos nuevos de cáncer para estimar la incidencia si no se cuenta con un registro poblacional, lo cual es común en muchos países. En lo referente a la mortalidad, el problema también es complicado; comúnmente se publican las defunciones que se presentan en el grupo de 5 a 14 años y en el de 15 a 24 años de edad, ${ }^{2}$ con lo cual se pierde la información referente al grupo de adolescentes. Por lo tanto, además de que existe poca información epidemiológica sobre el cáncer en este grupo de edad, la mayoría de los autores describen la frecuencia del cáncer en las primeras dos décadas de la vida sin hacer la diferencia entre niños y adolescentes, lo cual complica el análisis que sobre la incidencia y mortalidad por cáncer pueda realizarse en el grupo de adolescentes. ${ }^{3}$ De ahí la importancia de hacer un consenso para establecer el grupo de edad más apropiado que deberá considerarse para reportar los datos epidemiológicos de adolescentes. ${ }^{3,5}$

Por otra parte, debido a que el cáncer en adolescentes es diferente del infantil (menores de 14 años) y de los carcinomas de los adultos, algunos autores afirman que el patrón de cáncer entre adolescentes es un problema especial que requiere solución especial. $\mathrm{Su}$ etiología es pobremente entendida; algunos casos surgen como parte de un síndrome genético y otros pueden atribuirse a factores de riesgo ambientales. Asimismo, las necesidades clínicas de adolescentes con cáncer difieren de las de los niños y los adultos que tienen el mismo tipo de cáncer, y por tanto, requieren atención multidisciplinaria. ${ }^{4}$ La importancia de reconocer en forma independiente el cáncer en la adolescencia va aparejada a un conjunto de situaciones prácticas. Por ejemplo, los y las jóvenes adolescentes con cáncer suelen ser enviados a pabellones pediá- tricos para su atención. Esto a pesar de la evidente diferencia que existe, en diversos sentidos, entre estos dos grupos de pacientes. En otros casos, adolescentes con cáncer son enviados a salas oncológicas de adultos. Esta situación es claramente incómoda para los enfermos. El efecto del diagnóstico de cáncer para ellos es devastador; adaptarse a los efectos de la enfermedad y su tratamiento les es particularmente difícil. Pueden perder el control personal, es frecuente que presenten cambios en las relaciones personales, así como una gran incertidumbre y angustia ante el futuro. De ahí la conveniencia de reconocer las peculiaridades del cáncer en esta etapa de la vida.,3

A pesar de que el panorama es complicado y difícil, algunos pasos se han tomado en la dirección correcta. Recientemente, en diferentes partes del mundo se han establecido unidades de atención a adolescentes con cáncer. ${ }^{4}$ Inglaterra puede ser un ejemplo: en este país, el Primer Fideicomiso de Cáncer del Adolescente para atender las necesidades de este grupo de pacientes se estableció desde 1990, y su objetivo principal fue cubrir las necesidades de atención específica al adolescente con cáncer. Este mismo Fideicomiso contribuyó para que se llevara a cabo la Primera Conferencia Internacional sobre Cáncer en el Adolescente en 1994, y de ahí surgió la estimación que el Reino Unido necesita 20 de estas unidades para atender a aquellos que desarrollen alguna neoplasia. ${ }^{5}$

\section{Material y métodos}

Se revisó la literatura médica nacional e internacional para un periodo de 15 años (1985-1999), con el objetivo de obtener artículos que informaran sobre la epidemiología descriptiva del cáncer en adolescentes, para lo cual se utilizaron medios digitales (Medline) y se analizaron las características de tiempo, lugar y persona y las relaciones entre estas variables. Asimismo, y con el objetivo de revisar las características generales de las principales neoplasias en adolescentes, se obtuvieron artículos específicos para tal fin. Cabe señalar que las tasas que aquí se reportan estarán dadas por $10^{6}$ a reserva de que se especifique lo contrario. 


\section{Morbilidad y mortalidad entre adolescentes}

La reducción en la mortalidad infantil ha modificado las perspectivas de vida y por lo tanto la pirámide de la población. Existe un mayor número de sujetos jóvenes ya que aproximadamente $30 \%$ de la población corresponde a individuos entre 10 y 25 años de edad; este grupo de población reside principalmente en países en desarrollo. ${ }^{6}$

En la actualidad las principales causas de muerte en adolescentes de países como EUA son accidentes, principalmente automovilísticos, suicidio, homicidio, cáncer, enfermedades cardiovasculares y las anomalías congénitas. Si se consideran las primeras tres causas como una sola categoría el cáncer, entonces, ocupa la segunda posición; o bien, por la importancia que representa, exceptuando las violentas, el cáncer es la principal causa de muerte. ${ }^{7}$

\section{Incidencia del cáncer}

La información que hace referencia a la incidencia del cáncer en adolescentes no es precisa con relación a la edad; en la mayoría de las publicaciones sobre la incidencia no se especifica el grupo de edad de 10 a 19 años. En el ámbito mundial, la división por edades para describir la incidencia de cáncer está basada en grupos de 15 años (<15 años, 15 a 34, 35 a 49, etcétera), lo que dificulta conocer el panorama del cáncer en la adolescencia. ${ }^{8-11}$ De las publicaciones exclusivas acerca de los niños, se describen grupos de 5 hasta los 15 años, y para los adolescentes, generalmente se utiliza el intervalo de 10 a 14 años. ${ }^{11-18}$ Sólo algunos autores se han preocupado por dar a conocer los resultados de la incidencia del cáncer en todos los años que abarca la adolescencia, es decir, entre los 10 y 19 años de edad. ${ }^{19-22}$

En el cuadro I se presenta en forma global la incidencia del cáncer en la adolescencia; se puede observar la discrepancia que existe tanto para determinar la edad como para describir el tipo de neoplasia. La incidencia de los países anglosajones comparada con la de México es mucho mayor; sin embargo, es necesario señalar que el estudio de México fue retrospectivo y probablemente la incidencia está subestimada. Cuando se describe en forma independiente a los grupos de edad de 10 a 14 y 15 a 19, la incidencia es mucho más alta para el segundo grupo, incluso es mayor que la del grupo de menores de cinco años, que en la edad pediátrica es el grupo de mayor incidencia. ${ }^{22}$ Por tipo de tumor, al dividir la etapa de adolescencia en quinquenios, se encuentran diferencias en la incidencia de neoplasias; para EUA, en el grupo 10 a 14 años durante el periodo de 1986 a 1995, los tumores más frecuentes fueron los que se desarrollan en el sistema nervioso central (TSNC), seguidos por leucemias, linfomas y tumores óseos con tasas de $10^{6}$ de 24.6, 23.5, 22.0 y 12.4, respectivamente; mientras que para el grupo de 15 a 19 años, los más frecuentes fueron los linfomas, los tumores de células germinales (TCG), las leucemias y los TSNC (tasas de 47.8, 30.8, 21.4, y 20.2, respectivamente). Por lo señalado, puede considerarse que en la adolescencia la frecuencia de otros tumores como el retinoblastoma, los tumores renales, hepáticos y el neuroblastoma disminuye en forma considerable; pero otro como el melanoma maligno y el carcinoma de tiroides emergen como un problema especial en el grupo de 15 a 19 años. ${ }^{21,22}$

En el cuadro II se describen los resultados de la incidencia del cáncer en adolescentes de diferentes países, estratificando por sexo. En general, las consideraciones hechas en los párrafos anteriores son las mismas. Sin embargo, debe señalarse que la incidencia de cáncer es siempre mayor en el sexo masculino y que si bien, en ambos sexos, los TCG representan un rubro importante en los varones, en especial en el grupo de 15 a 19 años, en algunos países como Australia el cáncer de testículo, es el de mayor incidencia; cabe comentar que para las australianas adolescentes el cáncer de piel es el más frecuente. ${ }^{20}$ Por lo general, el cáncer de tiroides y de piel no se distinguen en

\begin{tabular}{|c|c|c|c|c|}
\hline INCIDENC & $\mathrm{CIA}{ }^{*} \mathrm{D}$ & $\begin{aligned} & \text { Cuac } \\
&= \text { CÁNCE } \\
& \text { IFERENT }\end{aligned}$ & $\begin{array}{l}\text { dro I } \\
\text { R EN ADOLESCENT } \\
\text { ES PAÍSES }\end{array}$ & ES EN \\
\hline & $\begin{aligned} & E \\
& 1986\end{aligned}$ & $\begin{array}{l}\text { A } \\
-1995\end{array}$ & $\begin{array}{c}\text { México } \\
1982-1991\end{array}$ & $\begin{array}{l}\text { Reino Unido } \\
\quad 1993\end{array}$ \\
\hline Tipo de tumor & $10-14$ & $15-19$ & $10-14$ & $13-19$ \\
\hline Todos & 117.3 & 181.3 & 96.4 & 112.0 \\
\hline Leucemia & 23.5 & 21.4 & 34.6 & 16.0 \\
\hline Linfomas & 22.0 & 47.8 & 19.6 & 20.0 \\
\hline$\overline{S N C}$ & 24.6 & 20.2 & 13.6 & 13.0 \\
\hline SN S & 0.8 & 0.5 & 1.5 & - \\
\hline Retinoblastoma & 0.0 & 0.1 & - & - \\
\hline Renales & 0.6 & 0.4 & 3.0 & - \\
\hline Hepáticos & 0.4 & 1.0 & - & - \\
\hline 0 seos & 12.4 & 14.0 & 9.0 & 10.0 \\
\hline Tejidos blandos & - & 15.9 & 6.0 & 6.0 \\
\hline Células germinales & 6.7 & 30.8 & 6.0 & 13.0 \\
\hline Carcinomas & 4.1 & 18.7 & 3.0 & 10.0 \\
\hline 0 tros & 2.8 & 10.5 & - & 16.0 \\
\hline
\end{tabular}

Fuente: referencias 3,13,22*Tasas x 106; EUA: Estados U nidos de América; SN C: Sistema N ervioso Central; SN S: Sistema N ervioso Simpático. 


\begin{tabular}{|c|c|c|c|c|c|c|c|c|c|c|c|c|c|c|c|c|}
\hline & & & INCIDE & ENCIA* & DE CÁN & $\begin{array}{r}\mathrm{Cu} \\
\text { NCER E }\end{array}$ & $\begin{array}{l}\text { uadro I } \\
\text { EN ADO }\end{array}$ & $\begin{array}{l}\text { II } \\
\text { LESCEI }\end{array}$ & NTES $S$ & SEGÚN & SEXO & & & & & \\
\hline & $\begin{array}{r}\text { EU } \\
1973-1\end{array}$ & $\frac{A}{1982}$ & 1968- & $\frac{\text { Dinama }}{1987}$ & $\frac{\operatorname{arca}}{1943-19}$ & & $\begin{array}{r}\text { Austra } \\
1972-1 \\
\text { Grup }\end{array}$ & $\begin{array}{l}\frac{\text { alia }}{1991} \\
\text { po de eda }\end{array}$ & $\begin{array}{r}\text { Méxic } \\
1992-1 \\
\text { ad y sexo }\end{array}$ & & $\begin{array}{c}\text { Franc } \\
1984-1\end{array}$ & & & $\frac{\text { onnecticut }}{1965-10}$ & $\frac{t}{t(E U A)}$ & \\
\hline Tipo de tumor & $\frac{\mathrm{H}}{10-14}$ & $\frac{M}{10-14}$ & $\frac{H}{10-19}$ & $\frac{M}{10-19}$ & $\frac{\mathrm{H}}{10-14}$ & $\frac{M}{10-14}$ & $\frac{\mathrm{H}}{10-19}$ & $\frac{M}{10-19}$ & $\frac{\mathrm{H}}{10-14}$ & $\frac{M}{10-14}$ & $\frac{\mathrm{H}}{10-14}$ & $\frac{M}{10-14}$ & $\frac{\mathrm{H}}{10-14}$ & $\frac{M}{10-14}$ & $\begin{array}{c}\mathrm{H} \\
15-19\end{array}$ & $\begin{array}{c}M \\
15-19\end{array}$ \\
\hline Todos & 112.3 & 99.5 & 148.8 & 109.7 & 101.7 & 88.2 & 158.4 & 140.0 & 108.8 & 82.0 & 98.5 & 87.4 & 101.2 & 93.9 & 179.9 & 176.9 \\
\hline Leucemia & 26.5 & 17.7 & 28.8 & 20.2 & 28.8 & 23.6 & 30.2 & 20.6 & 41.9 & 27.3 & 16.6 & 16.6 & 27.6 & 19.9 & 29.1 & 19.1 \\
\hline Linfomas & 26.8 & 18.8 & 30.2 & 19.0 & 18.6 & 11.7 & 28.9 & 17.4 & 29.9 & 9.1 & 25.6 & 10.1 & - & - & - & $\overline{-}$ \\
\hline SNC & 22.0 & 19.1 & 30.5 & 25.1 & 25.6 & 23.7 & 21.4 & 19.1 & 10.0 & 15.2 & 25.6 & 23.9 & 22.6 & 18.5 & 18.7 & 14.1 \\
\hline SN S & - & 1.5 & 2.2 & 1.6 & 2.0 & 1.0 & 1.1 & 0.9 & 3.0 & - & - & - & - & - & - & - \\
\hline Retinoblastoma & 0.1 & - & - & - & - & - & 0.2 & - & - & - & 0.9 & - & - & - & - & - \\
\hline Renales & 1.4 & 1.4 & 0.8 & 1.2 & 0.7 & 1.0 & 0.7 & 1.3 & - & - & 0.9 & - & 0.5 & 0.5 & 1.5 & - \\
\hline Hepáticos & - & - & 0.6 & 0.5 & 0.3 & 0.5 & 0.8 & 0.2 & - & - & 0.9 & - & - & - & - & - \\
\hline 0 seos & 12.6 & 11.7 & 13.3 & 8.2 & 10.3 & 9.9 & 16.5 & 10.8 & 12.0 & 6.1 & 8.7 & 11.0 & 9.0 & 8.5 & 15.4 & 11.1 \\
\hline Tejidos blandos & 7.6 & 7.7 & 7.5 & 6.2 & 6.5 & 4.7 & 9.5 & 8.3 & 3.0 & 9.1 & 10.5 & 7.4 & - & - & - & - \\
\hline Células germinales & - & - & 21.4 & 7.0 & 1.8 & 4.1 & 13.0 & 8.9 & 3.0 & 9.1 & 2.6 & 7.4 & - & - & - & - \\
\hline Carcinomas & - & - & 11.6 & 18.7 & 5.5 & 6.8 & 35.5 & 51.3 & 6.0 & - & 7.0 & 11.0 & - & - & - & - \\
\hline 0 tros & 14.0 & 21.5 & 1.8 & 2.0 & 1.8 & 1.2 & 0.8 & 1.4 & - & - & 0.9 & - & - & - & - & - \\
\hline & $9-21$ & & & & & & & & & & & & & & & \\
\hline
\end{tabular}

la edad pediátrica, como se hace en los adultos; esto es un punto que debe considerarse para el reporte de futuras estadísticas. En México no se tienen datos para el grupo de 15 a 19 años y en el de 10 a 14 años la incidencia es mayor en el sexo masculino, y sigue el patrón latinoamericano de neoplasias (leucemias, linfomas y TSNC) y cambia un poco en el femenino (leucemias, TSNC, linfomas). ${ }^{13}$ Las diferencias en la incidencia en general, y el tipo de cáncer entre los países, posiblemente obedece a factores de riesgo diferentes. En relación con adolescentes mexicanos probablemente se debe a que se evaluó el grupo de 10 a 14 años.

Al comparar el grupo de 10 a 14 años con el de 15 a 19, en relación con las neoplasias más frecuentes para cada grupo, se presentan cambios interesantes: en los más jóvenes (grupo de 10 a 14 años) predominan las leucemias (25\%), los TSNC (25\%) y los linfomas (18\%); en cambio en el grupo de mayor edad predominan los linfomas (25\%), los tumores epiteliales $(18 \%)$, los tumores óseos $(15 \%)$, las leucemias, los TSNC, y los TCG alcanzan la misma frecuencia (10\%). En relación con los TCG, particularmente los de testículo, tiende a incrementarse. ${ }^{3}$
En relación con la raza (cuadro III), según datos de EUA se observa que entre adolescentes blancos de 15 a 19 años la incidencia de cáncer fue 1.5 veces mayor que la de los adolescentes negros. En forma específica, según tipo de tumor, las diferencias más importantes se presentaron en el melanoma y el sarcoma de Ewing (la razón blancos/negros fue de 53.7 y 18.0 respectivamente); asimismo, la leucemia linfoblástica aguda fue dos veces más frecuente en los blancos y para los TCG, en donde también la mayor incidencia fue para adolescentes blancos; sólo en los varones hubo diferencia. ${ }^{22}$

En cuanto a la tendencia, en forma global, en los últimos años se ha documentado un incremento de la incidencia para adolescentes norteamericanos de 15 a 19 años. ${ }^{19}$ En la revisión de 1975 a 1995 del Surveillance Epidemiology and Results Program (SEER) en los EUA se encontró que la incidencia para este periodo fue de 183 y para el de 1990 a 1995 llegó a 203.8. ${ }^{22}$ Este incremento también se ha visto en los menores de 15 años, sin embargo, la incidencia en el grupo 15 a 19 años ha sido mucho mayor. Por otro lado, en el grupo de 15 a 19 años parece no existir cambio en la inciden- 


\section{Cuadro III \\ INCIDENCIA* DE CÁNCER EN ADOLESCENTES (15 a 19 años) de Estados Unidos de AméricA, SEGÚN RAZA, 1986-1995}

\begin{tabular}{lrrr}
\multirow{2}{*}{ Tipo de tumor } & \multicolumn{2}{c}{ Raza } & \\
& Blanca & Negra & Razón B/N \\
\cline { 2 - 3 } Total & 213.5 & 144.8 & 1.5 \\
\hline Leucemia linfo blástica aguda & 14.3 & 6.4 & 2.2 \\
\hline Leucemia mieloblástica aguda & 8.3 & 7.1 & 1.2 \\
\hline Enfermedad de Hodgkin & 36.5 & 26.9 & 1.4 \\
\hline Linfoma no Hodgkin & 16.1 & 9.4 & 1.7 \\
\hline Sistema nervioso central & 21.8 & 15.8 & 1.4 \\
\hline O steosarcoma & 9.2 & 8.4 & 1.1 \\
\hline Sarcoma de Ewing & 5.4 & 0.3 & 18.0 \\
\hline Sarcoma de partes blandas & 14.5 & 20.5 & 0.7 \\
\hline Tumor de células germinales & 33.9 & 13.8 & 2.5 \\
\hline Carcinoma de tiroides & 15.5 & 6.7 & 2.3 \\
\hline Melanoma & 16.1 & 0.3 & 53.7 \\
Fuente: referencia 22 & & & \\
* Tasas x 10 & & & \\
\hline
\end{tabular}

cia para los TSNC, el melanoma, el cáncer de tiroides, la enfermedad de Hodgkin o los sarcomas de tejidos blandos. ${ }^{22}$

Por último, cabe señalar que otra dificultad para la interpretación de las diferentes estadísticas sobre la incidencia del cáncer en adolescentes está dada porque, al describir a los mayores de 15 años, se utiliza la clasificación basada en la topografía y no en la histología. Por esta razón Fritschi y colaboradores ${ }^{20}$ en 1995 publicaron la comparación de una y otra clasificación en adolescentes de 10 a 19 años y observaron que la histológica provee un esquema más completo; sin embargo parece ser incompleto, puesto que en edades mayores se incluyen algunos tumores (carcinomas de colon, pulmón y glándulas salivales) propios de la edad adulta. Sugieren que debería haber una adaptación de la clasificación histológica exclusiva para adolescentes, ya que conforman un grupo más parecido a los niños que a los adultos.

\section{Mortalidad por cáncer}

La información relacionada con la mortalidad por cáncer en adolescentes es aún menor que para la incidencia. De la revisión efectuada, sólo se encontraron dos artículos que hacen referencia a tasas de mortalidad en el grupo de 10 a 14 años, uno de la Comunidad Europea y otro de México, los cuales están resumidos en el cuadro IV. ${ }^{15,18} \mathrm{Al}$ igual que la inciden-

\section{Cuadro IV \\ Mortalidad* POR CÁNCER EN ADOLEsCENTES DE 10 A 14 AÑOS}

\begin{tabular}{|c|c|c|c|c|}
\hline \multirow{3}{*}{ Tipo de la neoplasia } & \multicolumn{4}{|c|}{ País } \\
\hline & \multicolumn{2}{|c|}{$\begin{array}{c}\text { Comunidad europea } \\
(1979-1988) \\
\text { Sexo }\end{array}$} & $\begin{array}{c}\text { México } \\
\text { (1990-1994) }\end{array}$ & \multirow{2}{*}{$\begin{array}{l}\text { Sexo } \\
\text { Ambos }\end{array}$} \\
\hline & $M$ & $F$ & Tipo de neoplasia & \\
\hline Todas & 50.8 & 38.6 & Todas & 64.1 \\
\hline Leucemias & 21.4 & 15.3 & Leucemias & 38.2 \\
\hline Linfomas & 1.0 & 0.8 & Linfomas & 6.7 \\
\hline Enfermedad de Hodgkin & 4.9 & 2.1 & Enfermedad de Hodgkin & 4.4 \\
\hline Sistema nervioso central & 10.2 & 7.6 & Linfoma no Hodgkin & 2.3 \\
\hline 0 jo & 0.1 & 0.1 & Histiocitosis & 0.2 \\
\hline Riñón & 0.6 & 0.7 & Sistema nervioso central & 7.1 \\
\hline Hueso y tejidos blandos & 6.6 & 6.3 & Sistema nervioso simpático & 0.3 \\
\hline O vario & - & 0.9 & Retinoblastoma & 0.1 \\
\hline Testículo & 0.2 & - & Renales & 0.5 \\
\hline 0 tros sitios específicos & 4.1 & 3.1 & Hepáticos & 0.3 \\
\hline \multirow[t]{5}{*}{ Sitios no especificados } & 1.7 & 1.4 & 0 seos & 5.4 \\
\hline & & & Tejidos blandos & 2.3 \\
\hline & & & Células germinales & 0.9 \\
\hline & & & Carcinomas & 0.5 \\
\hline & & & No específicos & 1.7 \\
\hline
\end{tabular}

Fuente: referencias 15,18

* Tasas x 106; M: masculino; F: Femenino

cia, la mortalidad en los varones es mayor; la mayor mortalidad se observa para las leucemias, el TSNC y los tumores óseos.

La tendencia de la mortalidad ha sido hacia la disminución; para adolescentes europeos la tasa en el periodo de 1960 a 1964 fue de 24; y en el de 1985 a 1989 de 19;18 esta reducción en la mortalidad también se observó en adolescentes de EUA entre 1975 y 1995 para los grupos de 10 a 14 y 15 a 19 años. ${ }^{22,23} \mathrm{La}$ tendencia por tipo de cáncer es hacia la disminución y en especial para las leucemias. ${ }^{23}$ Es importante señalar que a la mortalidad por cáncer, tanto en el grupo de niños (grupo de 0 a 14 años), como en el de adolescentes, se la considera como un índice de la calidad de la atención médica que reciben; por lo tanto, cuando las tasas de mortalidad por cáncer no han disminuido, se puede pensar que la calidad en la atención médica no ha sido del todo adecuada. ${ }^{24,25}$

\section{Sobrevida}

Como en otras edades pediátricas, entre adolescentes existe un incremento en la sobrevida para todos 
los grupos de cáncer (cuadro V). ${ }^{22}$ Sin embargo, con la información que se tiene hasta el momento no es posible conocer si existen diferencias en la sobrevida de adolescentes, en comparación con el resto de la población pediátrica. Algunos autores consideran que los y las adolescentes tienen mejor pronóstico que los niños menores de 10 años, pero otros opinan que, por el hecho de no existir un consenso sobre la edad para incluir a adolescentes en los esquemas de tratamiento de adultos o niños, la sobrevida de los mayores de 15 años de edad es menor que en los menores de 15 años. ${ }^{26-28}$

La sobrevida de adolescentes con cáncer depende del tipo de cáncer que desarrollen. Debido al avance en la efectividad de la quimioterapia, las perspectivas para la mayoría de los pacientes con enfermedad de Hodgkin, con TCG o con sarcomas óseos son buenas; aun cuando en estos últimos sólo 50\% tiene una adecuada calidad de vida. Esto ha sucedido al mismo tiempo con el incremento exitoso de la cirugía reconstructiva de miembros, aunque su contribución a largo plazo en la calidad de vida aún no es seguro. También puede mencionarse que adolescentes con carcinoma de tiroides tienen un excelente pronóstico. Para varios tipos de cáncer, el objetivo actual es mantener la alta tasa de sobrevida en los subgrupos con un buen pronóstico con menos tratamientos tóxicos, mientras que

\section{Cuadro V}

SOBREVIDA EN ADOLESCENTES (15 a 19 AÑOS) DE Estados Unidos de América en dos periodos

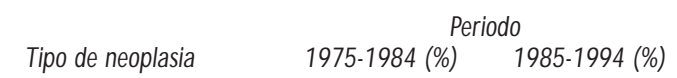

\begin{tabular}{lll} 
Total & 69 & 77 \\
\hline Leucemia linfo blástica aguda & 35 & 51 \\
\hline Leucemia mieloblástica aguda & 22 & 42 \\
\hline Enfermedad de Hodgkin & 88 & 90 \\
\hline Linfoma no Hodgkin & 56 & 69 \\
\hline Astrocitoma & 62 & 75 \\
\hline Meduloblastoma & 63 & 75 \\
\hline O steosarcoma & 49 & 59 \\
\hline Sarcoma de Ewing & 36 & 56 \\
\hline Sarcoma de partes blandas & 70 & 63 \\
\hline Rabdomiosarcoma & 40 & 45 \\
\hline Tumores de células germinales & 79 & 90 \\
\hline Carcinoma de tiroides & 99 & 99 \\
\hline Melanoma & 84 & 92 \\
\hline
\end{tabular}

Fuente: referencia 22 se intensifican los tratamientos para aquellos con datos de mal pronóstico. Esencialmente, los sobrevivientes deben mantenerse bajo vigilancia estricta y se les debe informar de los riesgos de futuras complicaciones, ya que el tratamiento puede afectar la fertilidad, puede desarrollar cánceres secundarios; en ocasiones, puede causar daño cardiovascular. Los efectos secundarios pueden ser tardíos y la mayoría son propios del efecto del tratamiento más que del cáncer per se. ${ }^{4}$

\section{A spectos específicos de algunas neoplasias en adolescentes}

Leucemias y linfomas. Se ha observado un incremento de la incidencia tanto para leucemias, linfomas no Hodgkin, osteosarcomas, y en forma muy importante para los TCG, en especial los de testículo; en estos últimos también se ha reportado un incremento en los adultos jóvenes. ${ }^{19}$

En relación con la incidencia del cáncer en adolescentes, se ha identificado una gran similitud entre los datos encontrados en Connecticut, Dinamarca y Nueva Gales del Sur. El 60\% fueron TSNC, leucemias y linfomas, principalmente. En los hombres predominaron las leucemias, los linfomas, sarcomas y TCG. En ambos géneros hubo una tendencia al incremento en los linfomas, principalmente a medida que eran mayores. Los tumores malignos de hueso, las neoplasias epiteliales y los TCG integraron otro 30\%. Algunas de las diferencias, como por ejemplo, que los TCG gonadales son cerca de la mitad de los que se encuentran en Dinamarca, han sido atribuibles a diferencias en los criterios de patología que se siguen en ambas naciones, más que a diferencias reales en las frecuencias observadas. ${ }^{21,29}$ Las tendencias observadas para los grupos de 10 a 14 y 15 a 19 años por Van Hoff y colaboradores $^{19}$ en Connecticut, EUA, entre 1935 y 1979, son también muy semejantes. Los factores de riesgo no difieren para los señalados en los niños de 0 a 14 años. ${ }^{22}$

En México, para el grupo de 10 a 14 años, la incidencia de leucemia linfoblástica aguda también se incrementó en el periodo de 1982 a 1991; en los varones fue de 3.9 para 1982 y de 14.6 para 1991, en las adolescentes se incrementó de 0.0 a $19.7 .{ }^{16}$ En relación con los linfomas, al igual que en adolescentes de EUA, el incremento de la incidencia de la enfermedad de Hodgkin ha sido mínimo; en el periodo señalado la incidencia varió entre 1.0 y 5.3. En lo que respecta a los linfomas no Hodgkin, la variación ha sido menor; para 1982 la incidencia fue de 2.0 y para 1991 de $4.9 .{ }^{17}$

Sistema nervioso central. De acuerdo con las estadísticas del Instituto Nacional del Cáncer de los EUA, la 
incidencia de los TSNC primarios entre los niños es de 30. Se ha identificado, sin embargo, que esta tasa está subestimada al no incluir neoplasias no malignas en la clasificación, y cuya importancia resalta porque el comportamiento de algunos tumores en el sistema nervioso central se considera como maligno, debido generalmente a las repercusiones sobre la morbilidad y mortalidad que ocasionan, dada su localización. De esta manera, la inclusión de tumores no malignos aumenta la incidencia de los TSNC en $28 \%$, es decir, la incidencia aumentó de 29.4 a 37.7. La contribución a este incremento es de $31 \%$ en la edad de 10 a 14 años, $y$ de $57 \%$ en los adolescentes de 15 a 19 años. ${ }^{30}$

A través de modelos mixtos log-lineal y Poisson se estudió la mortalidad de niños y adolescentes en España con tumores cerebrales malignos y su relación con factores socioeconómicos. La mortalidad se asoció positivamente con tres factores: la mortalidad infantil no relacionada con el cáncer, el porcentaje de superficie de tierra dedicada a la agricultura y las actividades industriales. El modelo final mostró que los estimadores ajustados de las tasas de mortalidad estandarizadas se incrementaban en $4 \%$ con cada aumento de $10 \%$ en el área dedicada a la agricultura, con $4 \%$ de exceso de riesgo para cada incremento de 1 por 1000 personas-año en la mortalidad infantil no relacionada con el cáncer, y con $7 \%$ de exceso de riesgo para categorías contiguas de actividad industrial. Concluyen que la mortalidad infantil puede ser un indicador de la calidad de la atención médica disponible; la mortalidad y el área destinada a la agricultura son una asociación ya conocida, y la actividad industrial puede considerarse como un factor de riesgo o como un marcador de otras condiciones asociadas con este tipo de tumores. ${ }^{31}$

Cáncer de tiroides. En Ucrania, durante los cinco años previos al accidente nuclear en Chernobyl, el cual ocurrió en 1986, se habían identificado 59 casos de cáncer de tiroides en el grupo de 0 a 18 años de edad; 25 de ellos en niños cuya edad era de 0 a 14 años, y 34 casos en adolescentes, con edad entre 15 a 18 años. Para los años de 1986 a 1997, el número de niños y adolescentes con cáncer de tiroides aumentó a 577, de los cuales 358 correspondieron a niños y 219 a adolescentes. El mayor número de casos se presentó en pacientes que vivían en áreas en donde las dosis de radiación se encontraban por arriba de los 0.50 Grays. ${ }^{32}$

Desde un punto de vista morfológico, los tumores tiroideos fueron de características agresivas; de tipo papilar en su mayor parte, aun cuando la mayoría mostraban un patrón de crecimiento papilar o sólido. Se encontró con mucha frecuencia metástasis a ganglios linfáticos, invasión venosa y diseminación fuera de la tiroides. Para la mayor parte de los pacientes el tratamiento requerido fue la tiroidectomía total con disección de ganglios linfáticos. ${ }^{32}$ También en la Baja Silesia se ha identificado un aumento en la incidencia de cáncer tiroideo durante los últimos 12 años, en pacientes de edades comprendidas entre 7 y 18 años. El cáncer tiroideo se observó con mayor frecuencia en pacientes con un solo nódulo y se identificó un alto grado de malignidad en los tumores analizados. ${ }^{33}$ Estos datos apoyan la hipótesis de que la radiación ionizante ambiental es uno de los principales factores de riesgo. ${ }^{23}$

Cáncer de colon. El cáncer de colon, uno de los tumores malignos más frecuentes entre los adultos, es raro en la adolescencia. La baja incidencia, asociada con síntomas no específicos del tumor y con una historia natural agresiva, es generalmente de mal pronóstico en los pacientes de esta edad..$^{34}$

Cáncer de laringe. En una revisión de 20 años de la literatura médica, se identificaron 47 casos de neoplasias malignas de la laringe en niños y adolescentes. La neoplasia más frecuente fue la variante embrionaria de rabdomiosarcoma. Los factores de riesgo que se han identificado son el tratamiento previo con radiación para la papilomatosis laríngea juvenil, la exposición intrauterina a la radiación ionizante y a los carcinógenos químicos. ${ }^{35}$ En Escocia, en hombres jóvenes se ha observado un incremento en la incidencia y mortalidad del cáncer de la cavidad oral y de laringe.

Cáncer pulmonar. En EUA se ha observado una disminución en la incidencia de cáncer pulmonar y en las tasas de mortalidad debidas a tal padecimiento. Sin embargo, las tendencias en el hábito tabáquico que se observan en adolescentes de ese país, hacen prever que las actuales tasas puedan aumentarse en un futuro próximo. $^{36}$

Cáncer mamario. El cáncer mamario es extremadamente infrecuente durante la adolescencia. ${ }^{37}$

Cáncer cervicouterino. Las infecciones por el virus del papiloma humano durante la adolescencia constituyen un factor de riesgo para el desarrollo de las lesiones intra-epiteliales escamosas de alto grado (LIEAG), así como de cánceres invasivos, lo que ha llevado a recomendar una vigilancia estrecha de las mujeres que inician relaciones sexuales a temprana edad. ${ }^{38}$

En mujeres adolescentes residentes en Nueva York que acudían a una clínica para infecciones de trasmisión sexual, y a quienes se les realizó un examen de 
Papanicolaou, se identificó una tasa de anormalidades en el frotis de $20.7 \%$, de los cuales $12.2 \%$ correspondió a células escamosas anormales de significado indeterminado, $7.7 \%$ a lesiones intraepiteliales escamosas de bajo grado de malignidad (LIEBG) y $0.7 \%$ a lesiones intraepiteliales de alto grado de malignidad (LIEAG). De este estudio se desprende que las adolescentes sexualmente activas se encuentran en alto riesgo de desarrollar anormalidades significativas en el frotis cervical, especialmente LIEBG. ${ }^{39}$ En Escocia se ha observado un aumento en las tasas de incidencia de cáncer cervical en las mujeres jóvenes. ${ }^{38}$

Sarcomas. En una serie de 26 pacientes con sarcomas de tejidos blandos no rabdomiosarcomatosos metastásicos (STBNR), se encontró que la edad promedio de presentación de la neoplasia fue 14.8 años. La sobrevida estimada a dos años para la cohorte fue de $34.6+/-8.9 \%$. La sobrevida a dos años libres de progresión fue de $15.4 \pm 6.3 \%$. Esta serie muestra que los niños y adolescentes con STBNR tienen mal pronósti$\mathrm{CO}$, similar al que se observa en los adultos. ${ }^{40}$

\section{Pronóstico social}

Los niños y adolescentes menores de 21 años de edad que presentaron algún cáncer entre 1970 y 1986, y que tuvieron una sobrevida mayor a cinco años fueron seguidos para determinar su estado civil. De 10425 sobrevivientes, $62 \%$ no se habían casado, $32 \%$ reportaron haberse casado y sólo $6 \%$ se habían divorciado. Comparados con la población de los EUA, los sobrevivientes blancos tuvieron una menor probabilidad para casarse, pero una vez casados fue menos probable que se divorciaran. En los sobrevivientes negros, en cambio, fue más probable que se hubieran casado y también más probable que se hubieran divorciado. Por tipo de cáncer, al comparar los sobrevivientes que desarrollaron un TSNC con los que tuvieron otro tipo de tumor o con la población general de EUA, se encontró una menor probabilidad para casarse; pero los que se casaron fue más probable que se divorciaran. ${ }^{41}$

Por último, debe considerarse que el tratamiento integral tanto de adolescentes y los adultos jóvenes con cáncer, plantea problemas difíciles y específicos, los cuales están relacionados con el consentimiento para la terapia, así como con el interés o preocupación psicosocial relevante a su integración exitosa dentro de la sociedad, y su transición a la vida adulta. ${ }^{27}$

\section{Conclusiones}

Con la información revisada, cabe puntualizar que los datos de la epidemiología del cáncer en adolescentes son limitados. El principal problema radica en la dificultad para obtener el número de casos nuevos de cáncer que se presentan en una población, o de los que se mueren por estas enfermedades en el grupo de edad considerado como adolescentes (grupo de 12 a 18 años de edad). Este problema se agudiza aún más en los países que no cuentan con un registro poblacional sobre cáncer; registro que cuando se tiene, además, debe ser confiable. De ahí que la mayoría de la información provenga de países desarrollados, y por ello muy poco se conoce de la epidemiología del cáncer en los adolescentes de países en desarrollo y que muy probablemente tengan aspectos propios, diferentes a los que hemos señalado, lo cual en sí es tema de investigación.

Por otra parte, puede señalarse en general que la incidencia de cáncer es mayor en adolescentes de 15 a 19 años (adolescencia tardía), en el sexo masculino y en la raza blanca. Existe, además, una tendencia de la incidencia al incremento; la cual es más fácil de observar que la que presentan los niños de 0 a 14 años de edad.

Por el contrario, la mortalidad por cáncer en adolescentes residentes en países desarrollados presenta una tendencia hacia la disminución en forma general y específica, según los diferentes grupos de tumores, lo cual va unido a un incremento de la sobrevida, situación que no se presenta en adolescentes (10 a 14 años) mexicanos.

La epidemiología del cáncer en el niño (menores de 15 años) es poco conocida, aspecto que es más notorio en adolescentes. De ahí la necesidad de una mayor y mejor investigación epidemiológica descriptiva y analítica en este grupo de edad. La Clasificación Internacional para Cáncer en Niños ${ }^{20}$ puede utilizarse para la clasificación del cáncer en adolescentes; sin embargo, deben realizarse algunas modificaciones porque éstos tienen una mayor frecuencia de carcinomas, lo cual es característico del cáncer en este grupo de edad.

\section{Referencias}

1. Monroy AL,Velasco L. Fecundidad en la adolescencia. Causas, riesgos y opciones. Washington, DC: O PS, 1988:3.

2. Secretaría de Salud. Mortalidad 1980-1996. México, D.F.: Dirección General de Estadística, 1998; 1:1-68.

3. Lewis IJ. Cancer in adolescence. Br Med Bull 1996;52(4):887-897.

4. Stiller C A. Cancer in adolescence. BMJ 1994;308:1382-1383.

5. Chambers EJ. C ancer and the adolescent. J R Soc Med 1997;90:176-177. 6. Daniel W A.Valoración de los adolescentes. En: Behrman RE, Vaughan VC, ed. N elson.Tratado de pediatría. 12ª ed. México, D.F.: N ueva Editorial Interamericana,1988:40-48.

7. National for Health Statistics. Health, United States, 1989 and 1993. H yattsville (MD): US G overnment Printing 0 ffice, 1994. [D H HS publication (PHS) 94-1232].

salud pública de méxico / vol.45, suplemento 1 de 2003 
8. D evesa SS, Blot W J, Stone BJ, Miller BA, Tarone RE, Fraument JF. Recent cancer trends in the United States. J N atl Cancer Inst 1995;87:175-182. 9. Swerdlow AJ, D os Santos-Silva I, Reid A, Q iao Z, Brewster DH,Arrundale T.Trends in cancer incidence and mortality in Scotland: Description and possible explanations. Br J Cancer 1998; 77 (Suppl 3):1-16.

10. Parkin DM, Pisani P, Ferlay J. Estimates of the worldwide incidence of 25 major cancers in 1990. Int J Cancer 1999;80:827-841.

11. Bernard JL, Bernard-C outeret E, C oste D, Thyss A, Scheiner C, Perrimond $\mathrm{H}$. Childhood cancer incidence in the South-East of France. A report of the Provence-Alpes-Cote d'Azur and Corsica Regions Pediatric Cancer Registry, 1984-1991. Eur J Cancer 1993;29A:2288-2291.

12. De N ully-Brown P, Hertz H, O Isen JH, Yssing M, Scheibel E, MollerJensen 0. Incidence of childhood cancer in Denmark 1943-1984. Int J Epidemiol 1989;18:546-555.

13. Fajardo-G utiérrezA, N avarrete-MartínezA, Reynoso-G arcía M,ZarzozaMorales ME, Mejía-A ranguré JM,Yamamoto-Kimura LT. Incidence of malignant neoplasms in children attending social security hospitals in Mexico City. Med Pediatr O ncol 1997;29:208-212.

14.Young JL, Ries LG, Silverberg E, Horm JW, Miller RW. C ancer incidence, survival, and mortality for children younger than age 15 years. Cancer 1986;58:598-602.

15. Fajardo-Gutiérrez A, Mejía-A ranguré JM, H ernández-C ruz L, Mendoza-Sánchez HF, Garduño-Espinosa J, Martínez-García MC. Epidemiología descriptiva de las neoplasias malignas en niños. Rev Panam Salud Publica 1999;6:75-88.

16. Mejía-A ranguré JM, Fajardo-Gutiérrez, Bernáldez-Ríos R, Farfán-Canto JM, 0 rtiz-Fernández A, Martínez-G arcía MC. Incidence trends of acute leukemia among the children of Mexico City: 1982-1991. Arch Med Res 1996;27:223-227.

17. Mendoza-Sánchez HF, Q uintana-Sánchez JA, Rivera-Márquez H, MejíaD omínguez AM, Fajardo-G utiérrez A. Epidemiology of lymphomas in children residing in Mexico City. Arch Med Res 1998;29:67-73.

18. Martos MC, $\mathrm{O}$ Isen JH. Childhood cancer mortality in the European Community, 1950-1989. Eur J Cancer 1993; 29A:1783-1789.

19.Van H off J, Schymura MJ, McC rea-C urnen MG. Trends in the incidence of childhood and adolescent cancer in Connecticut, 1935-1979. Med Pediatr Oncol 1988;16:78-87.

20. Fritschi L, C oates M, McC redie M. Incidence of cancer among N ew South W ales adolescents: W hich classification scheme describes adolescent cancer better? Int J Cancer 1995;60:355-360.

21. Martos MC, W inther J, $O$ Isen JH. Cancer among teenagers in Denmark, 1943-1987. Int J Cancer 1993;55:57-62.

22. Smith MA, Gurney JG, Ries LAG. Cancer among adolescents 15-19 years old. En: Ries LAG, Smith MA, G urney JG, Linet M,Tamra T, Young JL ed. C ancer incidence and survival among children and adolescents: $U$ nited States SEER Program 1975-1995. Bethesda, (MD): N ational Cancer Institutes, SEER Program, 1999 N IH Publication N 0. 99-4649: 157-164. 23. Ries LAG. C hildren cancer mortality. En: Ries LAG, Smith MA, Gurney $J G$, Linet M, Tamra T, Young JL, ed. Cancer incidence and survival among children and ado lescents: United States SEER Program 1975-1995, Bethesda, (MD): N ational Cancer Institutes, SEER Program, 1999 N IH Publication N 0. 99-4649: 165-170.

24. Draper GJ. Childhood cancer:Trends in incidence, survival and mortality. Eur J Cancer 1995;31A:653-654.
26. LaVecchia C, Levi F, Lucchini F, Lagiou P,Trichopoulos D, N egri E.Trends in childhood cancer mortality as indicators of the quality of medical care in the developed W orld. Cancer 1998;83:2223-2227.

26. N achman J, Sather HN , Buckley JD, G aynon PS, Steinhertz PG, Tubergen D G.Young adults 16-21 years of age at diagnosis entered on childrens cancer group acute lymphoblastic leukemia and acute myeloblastic leukemia protocols. Cancer 1993;71:3377-3385.

27. Reaman GH, Bonfiglio J, Krailo M, Tebbi CK, Leikin S, Ettinger R et al. Cancer in adolescents and young adults. Cancer 1993;71:3206-3209.

28. Bleyer WA, Murphy TH, Robison LL, Ross JA, Pollock BH, Severson RK et al. $N$ ational cancer clinical trials: C hildren have equal access; adolescents do not. J A dolesc Health 1997;21:366-373.

29. 0 sterlind A. Diverging trends in incidence and mortality of testicular cancer in Denmark, 1943-1982. Br J C ancer 1986;53:501-505.

30. G urney JG, W all DA, Jukich PJ, D avis FG. The contribution of nonmalignant tumors to CN S tumor incidence rates among children in the U nited States. Cancer Causes Control 1999; 10: 101-105.

31. Pollan M, López-A bente G, Aragonés N, Ruiz M. Malignant brain tumour mortality among children and adolescents: Geographical distribution in Spain. J N eurol Sci 1999; 163: 127-136.

32. Tronko MD, Bogdanova TI, Komissarenko IV, Epstein O V, O liynyk Kovalenko A, Likhtarev LA et al.Thyroid carcinoma in children and adolescents in U kraine after the Chernobyl nuclear accident-Statistical data and clinicomorphologic characteristics. Cancer 1999; 86: 149-156.

33. W asikowa R, Iwanicka Z, Zak T, Lukienczuk T, Sawicz-Birkowska K. Nodular goiter and thyroid carcinoma in children and adolescents in a moderate endemic area (Loer Silesia-Sudeten endemia) in the last twelve years.J Pediatr Endocrinol Metab 1999;12:645-652.

34. Shankar A, Renaut AJ,W helan J, Taylor I. Colorectal cancer in adolescents.Ann R Coll Surg Engl 1999; 81: 100-104.

35. Ferlito A, Rinaldo A, Marioni G. Laryngeal malignant neoplasms in children and adolescents. Int J Pediatr 0 torhinolaryngol 1999;49:1-14. 36.W ingo PA, Ries LAG, Giovino GA, Miller DS, Rosenberg HM, Shopland DR.Annual report to the nation to the status of cancer 1973-1996, with a special section on lung cancer and tobacco smoking. J $\mathrm{N}$ atl $\mathrm{C}$ ancer Inst 1999; 91: 675-690.

37. N einstein LS. Breast disease in adolescents and young women. Pediatr Clin N orth Am 1999; 46: 607-629.

38. Moscicki AB. Human papillomavirus infection in adolescents. Pediatr Clin N orth Am 1999; 46: 783-807.

39. Edelman M, Fox AS, A lderman EM, N eal W, Shapiro A, Silver EJ et al. Cervical Papanicolaou smear abnormalities in inner city Bronx adolescents-prevalence, progression, and immune modifiers. Cancer 1999; 87:184-189

40. Pappo AS, Rao BN , Jenkins JJ, Merchant T, Poquette CA, C ain A et al. Metastatic nonrhabdomyosarcomatous soft-tissue sarcomas in children and adolescents:The St. Jude Children's Research Hospital experience. Med Pediatr O ncol 1999;33:76-82.

41. Rauck A M, G reen D M, Yasui Y, Mertens A, Robison LL. Marriage in the survivors of childhood cancer: A preliminary description from the childhood cancer survivor study. Med Pediatr O ncol 1999; 33: 60-63. 